\title{
Synthesis of 1, 8-Naphthyridine Derivatives using Biodegradable Starch Sulfuric Acid as Heterogeneous Catalyst
}

\author{
Kadeer Md ${ }^{1}$, Dr. Ramakanth Pagadala ${ }^{2}$, Dr. Venkatesan $\mathrm{Kasi}^{3}$ and Dr. Ramesh Domala ${ }^{4}$ \\ ${ }^{1}$ Asst. Professor, CVR College of Engineering/H\&S Department (Chemistry), Hyderabad, India \\ Email: khadeerchem@gmail.com \\ ${ }^{2}$ Asst. Professor, CVR College of Engineering/H\&S Department (Chemistry), Hyderabad, India \\ Email: pagadalaramakanth@gmail.com \\ ${ }^{3}$ Asst. Professor, CVR College of Engineering/H\&S Department (Chemistry), Hyderabad, India \\ Email: venkippk@gmail.com \\ ${ }^{4}$ Asst. Professor, Department of Chemistry and Pharmaceutical Sciences, M G University, Nalgonda, India \\ Email: drdo.ramesh@gmail.com
}

\begin{abstract}
: 2-amino-3-cyano substituted 1,8-naphthyridines has been developed in the presence of starch sulfuric acid (SSA catalyst using aminopyridine, malononitrile, and aromatic aldehydes via Knoevenagel condensation and Michael addition followed by intramolecular cyclization. The SSA catalyst could selectively be converted amino pyridine into 1,8-naphthyridines with good yield in a short period of time at room temperature. This catalyst can be efficiently reused several times without loss of its sustainable activity.
\end{abstract}

Index Terms: Starch sulfuric acid, naphthyridines, biodegradable solid catalyst, heterogeneous acid catalyst

\section{INTRODUCTION}

Greener synthetic transformations in the synthesis of bioactive heterocyclic compounds under solvent-free conditions provide more advantageous to the research laboratories and pharmaceutical industry [1-3]. Green chemical methodologies have become the motivating strength for organic researchers to afford environmentally kind paths for the synthesis of heterocyclic compounds. Hence, the enhancement of these privileged biologically important organic compounds in a greater yield of products in shorter process time, and from easily obtainable starting materials in great demand. The objective of green chemistry is to eliminate the hazardous byproducts, prevention of waste and increase the yield of the target compound with a sustainable eco-friendly environment $[4,5]$. Bio-degradable catalysts had developed various environmentally benign protocols for organic synthesis [6-8]. Multicomponent one pot synthesis has great profit to the pharmaceutical industry with design and synthesis of organic molecules, where it can minimize time, cost and energy [9].

Heterocyclic compounds have a distinct place among pharmaceutically significant natural products and synthetic organic compounds [10-12]. Nitrogen heterocyclic compounds are abundant in nature and are of great importance to human life as they exist in many natural products such as alkaloids, hormones and vitamins [13-16]. The construction of naphthyridine analogs are huge importance in pharmacological, bio medicinal and synthetic chemistry.
The synthesis of nitrogenous aromatic substituted compounds such as 1,8-naphthyridine moieties has magnificent significance in medicinal research. Substituted 1,8-naphthyridines have attracted considerable attention to fertile field of research due to its various biological activities such as antimicrobial [17], antimalarial [18], antiinflammatory [19, 20], anticonvulsant [21], hypoglycemic [22], vasodilators [23], psychotropic properties [24], insecticide [25] muscle relaxant [26] anticancer [27], $\beta$ adrenergic blocking properties [28], anti-hypertensive [29]. In previous years, nucleophilic substitution reactions were extensively used for the synthesis of substituted 1,8naphthyridine catalyzed by $\mathrm{CoCl}_{2}$ in THF, $N, N, N^{\prime}, N^{\prime}$ tetrabromobenzene-1,3-disulfonamide [30] and various multi step catalyzed compounds which have more disadvantages like the toxicity of reagents, long reaction time, harsh reaction conditions such as high reaction temperatures, expensive, most of the reactions require tedious work-up procedures and recyclability of catalyst. Therefore, there is a need to develop a more efficient, simple and convenient protocol using a conservation catalyst.

Recently, the research being focused on the starch sulfuric acid (SSA) as solid heterogeneous catalyst due to its unique properties such as more efficient, economical, compatible and eco-friendly. The present study is intended to the synthesis of poly functional substituted 1,8naphthyridines using SSA as heterogeneous catalyst.

\section{RESUlts AND Discussion}

SSA is prepared by the addition of drop wise $0.5 \mathrm{~g}$ of chlorosulfonic acid $(4.5 \mathrm{mmol})$ to the 2.5 grams of starch and added for about $10 \mathrm{ml}$ of $\mathrm{n}$-hexane at $0^{\circ} \mathrm{C}$ (Figure 1). Stirred the solution for about 2 hours and filter the compound. This SSA is white colored, non-hygroscopic solid and stable under reaction conditions. 


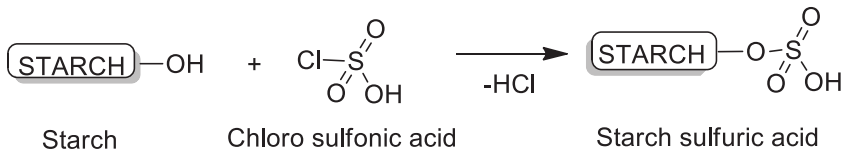

Figure 1. Synthesis of Starch sulfuric acid.

The Knoevenagel and Michael reactions are preliminarily examined by cyclocondensation of 2-aminopyridine, benzaldehyde and malononitrile in the presence of SSA (Figure 3), the reaction mixture was stirred at room temperature for about 30 minutes to get the desired product. To optimize the reaction conditions we conducted this reaction in various solvents such as acetonitrile, water and ethanol in which acetonitrile shows the best choice. Product was not formed without SSA catalyst (Table 1, entry 3) while in the presence of SSA $(5 \mathrm{mg})$, under the same conditions yield increased to $40 \%$ (Table 1 , entry 6 ). The yield of 1,8-naphthyridine increased with increasing the amounts of the catalyst from $5 \mathrm{mg}$ to $20 \mathrm{mg}$ (Table 1, entry 8). When the $30 \mathrm{mg}$ of SSA was used, there was no much difference in the product yield (Table 1, entry 9). $20 \mathrm{mg}$ of SSA was sufficient to catalyze the reaction effectively. All further studies were carried out under acetonitrile conditions with $20 \mathrm{mg}$ of catalyst at room temperature. In the end, SSA was washed with water and ethanol and dried at $70^{\circ} \mathrm{C}$ for one day. The isolated catalyst was reused in the next runs.

After optimization of the exemplary reaction, the structural variations in the aldehydes have no significant effect on the yield with either electron-withdrawing or electron-donating substituents bearing sensitive functional groups like $\mathrm{Cl}, \mathrm{NO}_{2}$ and $\mathrm{OCH}_{3}$. Different aromatic aldehydes were carried out the reaction with 2aminopyridines and malononitrile to afford the corresponding products with good yields. Notably, steric and electronic differences in the substrates have little effect on the rate and yield of the formed products (Table 2). In the projected reaction mechanism (Figure 2), SSA endures the Knoevenagel condensation reaction of aldehyde and after subsequent Michael addition of 2-aminopyridine gives intermediate. Finally, proton transfer and sequential intramolecular nucleophilic addition reaction, tautomerization and oxidation generate the 1,8naphthyridine derivatives.

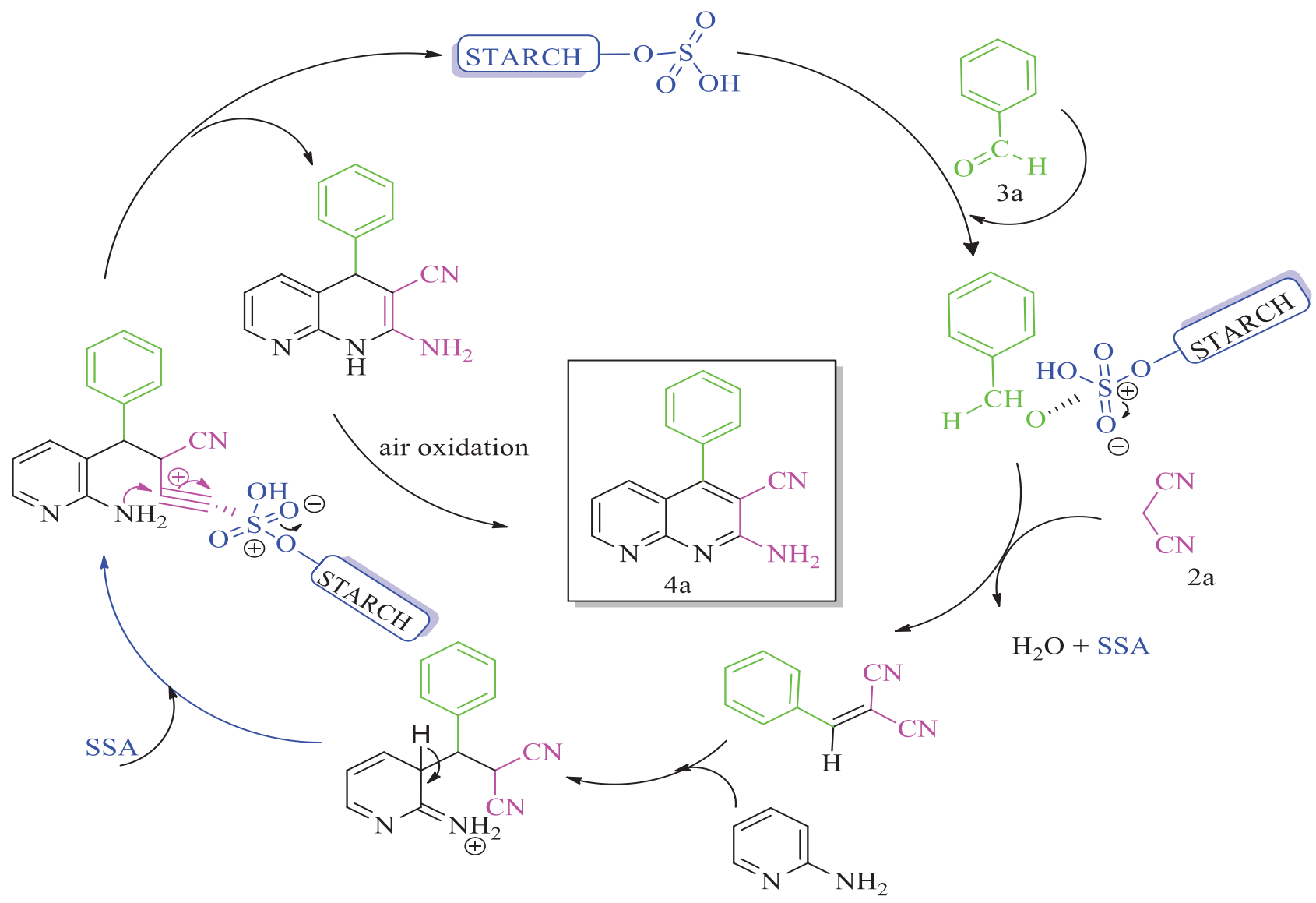

$1 \mathrm{a}$

Figure 2. Plausible reaction mechanism for the formation of 1,8-naphthyridines 


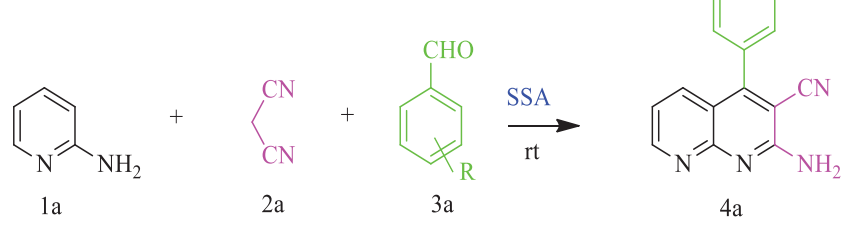

Figure 3. SSA catalyzed synthesis of the 1,8-Naphthyridines (4a-h).

\section{EXPERIMENTAL PROCEDURE}

TABLE I.

EFFECT OF SOLVENT ON SYNTHESIS OF 1,8-NAPHTHYRIDINES

\begin{tabular}{|c|c|c|c|c|c|c|}
\hline ENTRY & $\begin{array}{c}\text { Compo } \\
\text { und }\end{array}$ & $\begin{array}{c}\text { Amo } \\
\text { unt }\end{array}$ & Solvent & Temp & Time & $\begin{array}{c}\text { Yield } \\
\text { a }(\mathbf{0})\end{array}$ \\
\hline $\mathbf{1}$ & $\mathbf{4 a}$ & - & Ethanol & $\mathrm{rt}$ & $6 \mathrm{~h}$ & $\mathrm{~b}$ \\
\hline $\mathbf{2}$ & $\mathbf{4 a}$ & - & Ethanol & $70^{\circ} \mathrm{C}$ & $6 \mathrm{~h}$ & $\mathrm{~b}$ \\
\hline $\mathbf{3}$ & $\mathbf{4 a}$ & - & Acetonitrile & $\mathrm{rt}$ & $6 \mathrm{~h}$ & $\mathrm{~b}$ \\
\hline $\mathbf{4}$ & $\mathbf{4 a}$ & - & Water & $\mathrm{rt}$ & $6 \mathrm{~h}$ & $\mathrm{~b}$ \\
\hline $\mathbf{5}$ & $\mathbf{4 a}$ & - & Water & $80^{\circ} \mathrm{C}$ & $6 \mathrm{~h}$ & $\mathrm{~b}$ \\
\hline $\mathbf{6}$ & $\mathbf{4 a}$ & $5 \mathrm{mg}$ & Acetonitrile & $\mathrm{rt}$ & $6 \mathrm{~h}$ & 40 \\
\hline $\mathbf{7}$ & $\mathbf{4 a}$ & $20 \mathrm{mg}$ & Water & $\mathrm{rt}$ & $4 \mathrm{~h}$ & $\mathrm{~b}$ \\
\hline $\mathbf{8}$ & $\mathbf{4 a}$ & $20 \mathrm{mg}$ & Acetonitrile & $\mathrm{rt}$ & $30 \mathrm{~min}$ & 85 \\
\hline $\mathbf{9}$ & $\mathbf{4 a}$ & $30 \mathrm{mg}$ & Acetonitrile & $\mathrm{rt}$ & $30 \mathrm{~min}$ & 84 \\
\hline
\end{tabular}

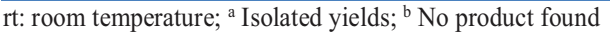

Typical procedure for the synthesis of 1,8-naphthyridine derivatives catalyzed by SSA $(4 a-h)$

A mixture of 2-aminopyridine (1 mmol), malononitrile (1 $\mathrm{mmol})$, benzaldehyde $(1.2 \mathrm{mmol})$, and SSA catalyst $(20 \mathrm{mg})$ in acetonitrile $(5 \mathrm{~mL})$ was stirred at room temperature for the appropriate length of time (Table 1, entry 6). The progress of the reaction was monitored by TLC (6:4, nhexane/ethyl acetate). After completion of the reaction, acetonitrile $(10 \mathrm{~mL})$ was added. The solid was filtered, washed with acetonitrile and dried. The residue was recrystallized from ethanol to afford the pure product. After evaporation of acetonitrile by reduced pressure, dichloromethane was added and filtered the catalyst. The collected SSA catalyst was reused four times in the same reaction.

\section{Product $4 a$}

Off-white solid, Mp. 149-152 ${ }^{\circ} \mathrm{C}$; IR (vmax, $\left.\mathrm{cm}^{-1}\right)(\mathrm{KBr})$ : 3249, 3329, 2219; NMR ${ }^{1} \mathrm{H}\left(300 \mathrm{MHz}, \mathrm{CDCl}_{3}\right): \delta 7.09$ (s, 2H, $\mathrm{NH}_{2}$ ), 7.14 (m, 1HAr), 7.17 (m, 2HAr), 7.20 (m, 2HAr), 7.24 (m, 1HAr), 7.26 (m, 1HAr), 7.28 (m, 1HAr); C-13 NMR (100 MHz, $\left.\mathrm{CDCl}_{3}\right)$ : $\delta 117.8,119.1,124.2,126.4$, $127.8,128.6,129.5,132.2,136.1,138.9,143.5,154.4$, 158.9, 169.9, 172.5; EIMS: (m/z): $242(\mathrm{M}+1)$; Anal. Theoretical for $\mathrm{C}_{15} \mathrm{H}_{10} \mathrm{~N}_{4}: \mathrm{C} ; 73.16, \mathrm{H} ; 4.09, \mathrm{~N} ; 22.75$. Found (Exp.): C; 73.20, H; 4.10, N; 22.79.
TABLE II.

SYNTHESIS OF 1,8-NAPHTHYRIDINES

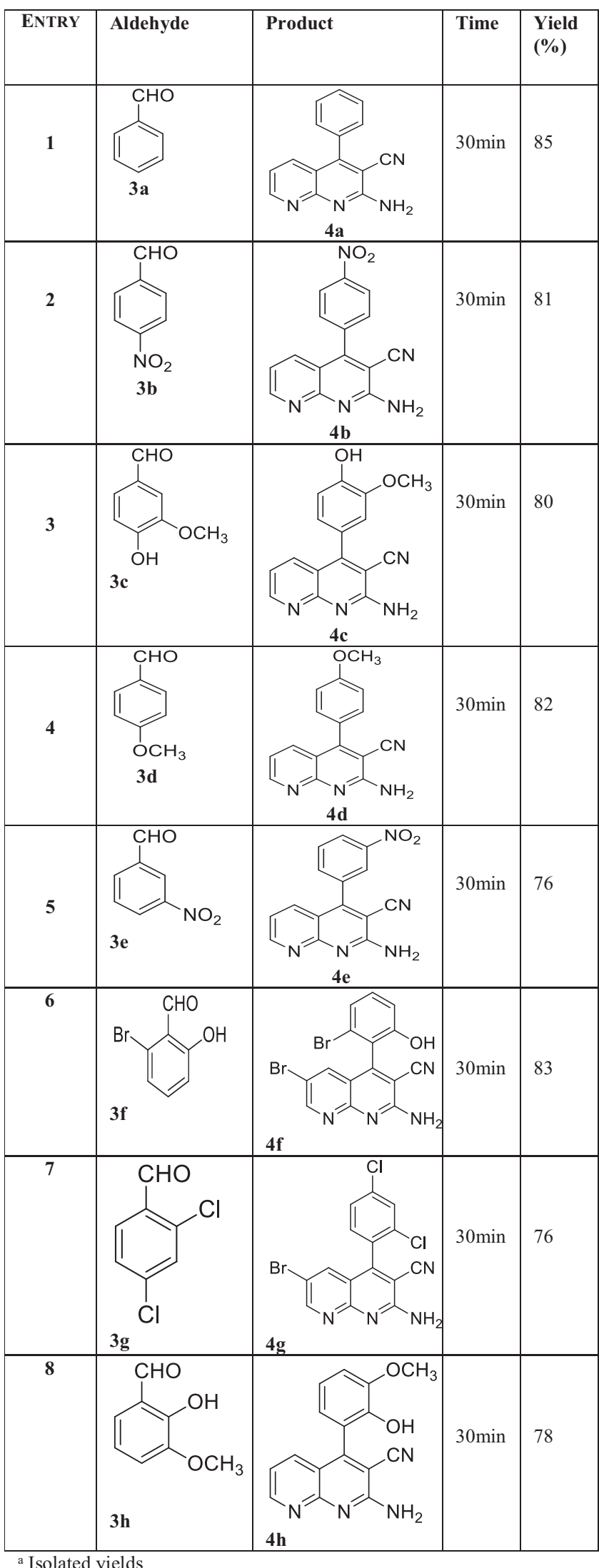

Product $4 b$ 
Off-white solid, Mp. 158-160 ${ }^{\circ} \mathrm{C}$; IR (vmax, $\left.\mathrm{cm}^{-1}\right)(\mathrm{KBr})$ : 3263, 3325,2223; NMR ${ }^{1} \mathrm{H}\left(300 \mathrm{MHz}, \mathrm{CDCl}_{3}\right): \delta 7.13$ (s, 2H, $\mathrm{NH}_{2}$ ), 7.18 (m, 2HAr), 7.24 (m, 2HAr), 7.29 (m, 1HAr), 7.30 (m, 1HAr), 7.36 (m, 1HAr); C-13 NMR (100 MHz, $\left.\mathrm{CDCl}_{3}\right): \delta 110.8,113.9,116.4,118.2,121.2,125.1,128.2$, 132.6, 135.9, 139.1, 141.8, 146.8, 147.6, 151.2, 155.5, 164.1; EMIS: (m/z): $292.32(\mathrm{M}+1)$ Anal. Theoretical for $\mathrm{C}_{15} \mathrm{H}_{9} \mathrm{~N}_{5} \mathrm{O}_{2}$ : C; 61.85, H; 3.11, N; 24.04, O; 10.99. Found (Exp.): C; 61.71, H; 3.10, N; 24.05, O; 10.96.

\section{Product 4c}

Off-white solid; NMR ${ }^{1} \mathrm{H}\left(300 \mathrm{MHz}, \mathrm{CDCl}_{3}\right): \delta 4.21(\mathrm{~s}$, $3 \mathrm{H}), 5.58(\mathrm{~s}, 2 \mathrm{H}), 6.11(\mathrm{~s}, 1 \mathrm{H}), 6.61(\mathrm{~d}, 1 \mathrm{H}), 6.70(\mathrm{~d}, 1 \mathrm{H})$, $7.17(\mathrm{~d}, 1 \mathrm{H}), 7.78(\mathrm{~m}, 3 \mathrm{H}), \mathrm{C}-13 \mathrm{NMR}\left(100 \mathrm{MHz}, \mathrm{CDCl}_{3}\right): \delta$ $62.5,88.4,115.0,117.8,118.9,121.7,125.1,128.7,132.8$, 135.6, 138.8, 141.4, 146.6, 149.3, 154.9, 164.5. EMIS: $(\mathrm{m} / \mathrm{z}): 293.16[\mathrm{M}+]$; Anal. Theoretical for $\mathrm{C}_{16} \mathrm{H}_{12} \mathrm{~N}_{4} \mathrm{O}_{2}$ : C; 65.75, H; 4.14, N; 19.17, O; 10.95. Found (Exp.): C; 65.74, $\mathrm{H} ; 4.09, \mathrm{~N} ; 19.15, \mathrm{O} ; 10.96$.

\section{Product $4 d$}

Off-white solid Mp. $155-157{ }^{\circ} \mathrm{C}$; IR (vmax, $\left.\mathrm{cm}^{-1}\right)(\mathrm{KBr})$ : 3240, 3292, 2231; NMR ${ }^{1} \mathrm{H}\left(300 \mathrm{~Hz}, \mathrm{CDCl}_{3}\right): \delta 3.749$ (s, $3 \mathrm{H}), 6.81(\mathrm{~m}, 2 \mathrm{H}, \mathrm{HAr}), 7.21(\mathrm{~m}, 1 \mathrm{H}, \mathrm{ArH}), 7.29(\mathrm{~m}, 1 \mathrm{H}$, ArH), $7.31\left(\mathrm{~s}, 2 \mathrm{H}, \mathrm{NH}_{2}\right), 7.37(\mathrm{~m}, 2 \mathrm{H}, \mathrm{ArH}), 7.40(\mathrm{~m}, 1 \mathrm{H}$, $\mathrm{ArH})$; C-13 NMR (100 MHz, $\left.\mathrm{CDCl}_{3}\right)$ : $\delta 111.9,117.0,121.6$, $123.4,125.0,128.1,130.1,134.2,135.8,141.8,145.9$, 147.1, 150.3, 151.8, 158.1, 168.0; EIMS: (m/z): $261(\mathrm{M}+)$. Anal. Theoretical for $\mathrm{C}_{16} \mathrm{H}_{12} \mathrm{~N}_{4}$ : C; 73.83, H; 4.65, N; 21.52 . Found (Exp.): C; 73.80, H; 4.63, N; 21.48.

\section{Product 4e}

Yellow solid, Mp. 159-161 ${ }^{\circ} \mathrm{C}$; IR $\left(\operatorname{vmax}, \mathrm{cm}^{-1}\right)(\mathrm{KBr})$ : 3247, 3309, 2231; NMR ${ }^{1} \mathrm{H}\left(400 \mathrm{MHz}, \mathrm{CDCl}_{3}\right): \delta 7.16(\mathrm{~s}$, $\left.2 \mathrm{H}, \mathrm{NH}_{2}\right), 7.21(\mathrm{~s}, 1 \mathrm{H}, \operatorname{ArH}), 7.23(\mathrm{~m}, 1 \mathrm{H}, \operatorname{ArH}), 7.24$ (m, 1H, ArH), 7.27 (m, 1H, ArH), 7.30 (m, 1HAr), 7.33 (m, 1H, $\mathrm{ArH}), 7.36$ (m, 1H, ArH); C-13 NMR (100 MHz, $\left.\mathrm{CDCl}_{3}\right): \delta$ $111.1,111.9,113.4,117.8,122.0,124.5,128.8,134.0$, $136.1,138.9,142.0,147.0,149.0,151.1,155.0,165.1$; EIMS: (m/z): $291.41(\mathrm{M}+)$. Anal. Theoretical for $\mathrm{C}_{15} \mathrm{H}_{9} \mathrm{~N}_{5} \mathrm{O}_{2}$ : C; 61.85, H; 3.11, N; 24.04, O; 10.99. Found: C; 69.82, H; 3.11, N; 24.01, O; 11.01.

\section{Product $4 f$}

Orange solid; mp $164-167^{\circ} \mathrm{C}$; IR (vmax, $\left.\mathrm{cm}^{-1}\right)(\mathrm{KBr}) 3423$, 3056, 2176, 1634, 1618, 1601, 1539, 1459, 1363, 1268, 1143, 1087; NMR ${ }^{1} \mathrm{H}\left(300 \mathrm{MHz}, \mathrm{CDCl}_{3}\right): \delta 6.92-7.47(\mathrm{~m}$, $3 \mathrm{H}, \operatorname{ArH}), 8.62$ (s, 1H, ArH), 8.67 (s, 1H, ArH), 7.73 (d, 2H, $\left.\mathrm{NH}_{2}\right), \delta 3.34$ (s, 1H, OH); C-13 NMR (100 MHz, $\left.\mathrm{CDCl}_{3}\right): \delta$ : $113.7,114.3,119.2,121.8,129.4,136.6,142.2,151.1$, 154.6, 159.8, 161.8. EIMS: (m/z): $420.06(\mathrm{M}+3)$ Anal. Theoretical for $\mathrm{C}_{15} \mathrm{H}_{8} \mathrm{Br}_{2} \mathrm{~N}_{4} \mathrm{O}: \mathrm{C} ; 42.89 .16, \mathrm{H} ; 1.89, \mathrm{~N}$; 13.84. Found (Exp.): C; 43.10, H; 1.87, N; 13.79.

\section{Product $4 g$}

Off-white solid; mp 164-166 ${ }^{\circ} \mathrm{C}$; IR $\left(\operatorname{vmax}, \mathrm{cm}^{-1}\right)(\mathrm{KBr})$ 3434, 3092, 1725, 1689, 1604, 1576, 1543, 1434, 1423, 1296, 1214, 1178, 1109; NMR ${ }^{1} \mathrm{H}\left(300 \mathrm{MHz}, \mathrm{CDCl}_{3}\right): \delta$ 7.56 (d, 2H, $\left.\mathrm{NH}_{2}\right), 7.75$ (s, $\left.1 \mathrm{H}, \operatorname{ArH}\right), 7.43$ (d, $J=7.2 \mathrm{~Hz}$, $1 \mathrm{H}, \mathrm{ArH}), 7.56(\mathrm{~d}, J=6.2 \mathrm{~Hz}, 1 \mathrm{H}, \mathrm{ArH}), 8.59$ (s, 1H, ArH), $8.63(\mathrm{~s}, 1 \mathrm{H}, \mathrm{ArH}) ; \mathrm{C}-13 \mathrm{NMR}\left(100 \mathrm{MHz}, \mathrm{CDCl}_{3}\right): \delta \mathrm{c}$ : $119.7,121.6,126.9,129.0,131.7,132.9,134.3,134.5$, 135.1, 135.7, 138.6, 139.4, 140.6, 142.1, 151.2,159.8, 160.1, 162.5. EIMS: $(\mathrm{m} / \mathrm{z}): 394(\mathrm{M}+)$, Anal. Theoretical for $\mathrm{C}_{15} \mathrm{H}_{7} \mathrm{BrCl}_{2} \mathrm{~N}_{4}$ : C; 45.72.16, H; 1.79, $\mathrm{N} ; 14.22$. Found (Exp.): C; 45.67, H; 1.80, N; 14.18 .

\section{Product $4 h$}

Orange solid; mp 167-169 ${ }^{\circ} \mathrm{C}$; IR (vmax, $\mathrm{cm}^{-1}$ ) (KBr) 3423, 3312, 3009, 2957, 1728, 1678, 1608, 1578, 1472, 1266, 1217, 1090, 865; NMR ${ }^{1} \mathrm{H}\left(300 \mathrm{MHz}, \mathrm{CDCl}_{3}\right): \delta 3.27$ (s, $1 \mathrm{H}, \mathrm{OH}), 3.59\left(\mathrm{~s}, 3 \mathrm{H}, \mathrm{OCH}_{3}\right), 7.71\left(\mathrm{~s}, 2 \mathrm{H}, \mathrm{NH}_{2}\right), 6.76(\mathrm{t}, \mathrm{J}=$ $5.0 \mathrm{~Hz}, 1 \mathrm{H}, \mathrm{ArH}), 6.84(\mathrm{~d}, \mathrm{~J}=6.1 \mathrm{~Hz}, 1 \mathrm{H}, \operatorname{ArH}), 7.07-7.21$ (m, 3H, ArH), 7.89 (t, J = $3.3 \mathrm{~Hz}, 1 \mathrm{H}, \mathrm{ArH})$; C-13 NMR $\left(100 \mathrm{MHz}, \mathrm{CDCl}_{3}\right): \delta 54.8,57.3,109.2,112.7,115.4,118.7$, $120.3,121.4,123.5,124.3,125.3,138.2,140.1,146.9$, 149.4, 149.7, 158.7, 163.2. EIMS: (m/z): $292.2(\mathrm{M}+)$, Anal. Theoretical for $\mathrm{C}_{16} \mathrm{H}_{12} \mathrm{O}_{2} \mathrm{~N}_{4}$ : C; 65.75.16, H; 4.14, N; 19.17 . Found (Exp.): C; 65.76, H; 4.10, N; 19.09.

\section{CONCLUSIONS}

In summary, we have synthesized a simple and effective method for the synthesis of medicinally important 2-amino 3-cyano substituted 1,8-naphthyridine derivatives. The SSA a catalyst reported in this paper will contribute positively to the green synthetic process by reducing the use of organic solvents. Catalytic amount of heterogeneous catalyst can be easily recovered and reutilized. The proposed method offers eco-friendly and reutilized SSA catalyst with easily available starting materials. In the view of ecological criteria, the present protocol should be a valuable addition for the synthesis of 2-amino 3-cyano substituted 1,8naphthyridines.

\section{REFERENCES}

[1] G. L. Thomas, R. J. Spandl, F. G. Glansdorp, M. Welch, A. Bender, J. Cockfield, J. A. Lindsay, C. Bryant, D. F. J. Brown, O. Loiseleur, H. Rudyk, M. Ladlow, D. R. Spring, Angew. Chem. Int. Ed., 2008, 47, 2808.

[2] A. E. Rosamilia, C. R. Strauss, J. L. Scott, Pure Appl. Chem. 2007, 79, 1869.

[3] J. M. Mitchell, J. T. Shaw, Angew. Chem. Int. Ed. 2006, 45, 1722.

[4] M. B. Gawande, V. D. B. Bonifacio, R. Luque, P. S. Brancoa and R. S. Varma, Chem. Soc. Rev., 2013, 42, 5522.

[5] M. B. Gawande, P. S. Branco and R. S. Varma, Chem. Soc. Rev., 2013, 42, 3371.

[6] R. Ramachandran, S. Jayanthi, Y. T. Jeong, Tetrahedron., $2012,68,363$.

[7] R. G. Koduri1, R. Pagadala1, R. Varala, CVR Journal of Science and Technology, 2018, 14, 107.

[8] M. D. Carrigan, K. J. Eash, M. C. Oswald, R. S. Mohan, Tetrahedron Lett., 2001, 42, 8133.

[9] J. C. Wasilke, S. J. Obrey, R. Tom Baker and G. C. Bazan, Chem. Rev., 2005, 105, 1001.

[10] R. Pagadala, S. Maddila, S. Rana, S.B. Jonnalagadda., RSC Adv., 2014, 4, 6602.

[11] R. Pagadala, S. Maddila, S.B. Jonnalagadda, Green Chem. Lett. Rev., 2014, 7, 131.

[12] Dr. K. Venkatesan, Dr. P. Ramakanth, Dr. Ch. Anjaneyulu, CVR Journal of Science and Technology, 2020, 18, 144. 
[13] R. Pagadala, S. Maddila, S. B. Jonnalagadda, Ultrasonics Sonochem., 2014, 21, 472.

[14] R. Pagadala, S. Maddila, V.D.B.C. Dasireddy, S.B. Jonnalagadda, Catal. Commun., 2014, 45, 148.

[15] R. Pagadala, S. Maddila, S.B. Jonnalagadda, Org. Prep. Proc. Int., 2014, 46, 261.

[16] R. Pagadala, S. Maddila, V. Moodley, W.E. van Zyl, S. B. Jonnalagadda, Tetrahedron Lett., 55 (2014) 4006.

[17] F. A. Ashour, S. A. Al-Mazoroa, J Pharm Sci., 1990, 4, 29.

[18] P. Melnyk, V. Leroux, C. Sergheraert, P. Grellier, Bioorg. Med. Chem. Lett., 2006, 16, 31.

[19] M. Amir, S. Shahani, Indian J Heterocyclic Chem., 1998, 8, 107.

[20] R. Paprocka, M. Wiese, A. Eljaszewicz, A. Helmin-Basa, A. Gzella, B. Modzelewska-Banachiewicz, J. Michalkiewicz, Bioorg. Med. Chem. Lett., 2015, 25, 2664.

[21] J. R. Dimmock, S. C. Vashishtha, J. P. Stables, Eur. J. Med. Chem., 2000, 35, 241.

[22] F. Kurzer, Org. Compd. Sulphur Selenium Tellurium, 1974, 4, 417.
[23] L. C. Cullen, S. H. Lalgudi, D. D. Feng Chi, P. Ashok, J. Comb. Chem. 2008, 10, 28.

[24] M. K. Khalid, S. Salman, S. Muhammad, T. Muhammad, M. S. Syed, P. Shahnaz, M. Iqbal Choudhar, Bioorg. Med. Chem., 2014, 22, 6509.

[25] A. A. Fadda, E. M. Afsah, S. Bondock, M. M. Z. Hammouda, B. Naturforsch, J. Chem. Sci., 2015, 70, 385.

[26] M. H. Sherlock, J. J. Kaminsky, W. C. Tom, J. F. Lee, S. C. Wong, R. W. Bryant, A. T. McPhail, J. Med. Chem. 1988, 31 , 2108.

[27] P. L. Ferrarini, C. Mori, G. Biogi, O. Livi, I. Tonetti, J. Heterocyclic Chem., 1984, 21, 417.

[28] P. L. Ferrarini, C. Mori, M. Badawneh, V. Calderone, R. Greco, C. Manera, A. Martinelli, P. Nieri, G. Saccomanni, Eur. J. Med. Chem., 2000, 35, 815.

[29] M. Ferrarini, M. Clendio, U. Calder, Ind. J. Chem., 2001, 20B, 619.

[30] G. Robert, D. S. Ziegler, C. Denise, A. C. Jakowetz, F. Auras, T. Bein, P. Knochel, Org. Lett., 2017, 19, 6384. 